\title{
紫水鸡冬季受食地选择
}

\author{
胡军华 $1,2,3,4$, 胡慧建 ${ }^{2, *}$, 杨道德 ${ }^{3}$, 蒋志刚 ${ }^{1, *}$ \\ (1. 中国科学院动物研究所 动物生态与保护生物学重点实验室, 北京 100101 ; \\ 2. 华南濒危动物研究所 广东省野生动物保护与利用公共实验室, 广州 510260 ; \\ 3. 中南林业科技大学 长沙, 410004; 4. 中国科学院研究生院, 北京 100049)
}

摘要: 2006 年 11 月 -2007 年 2 月, 在广东海丰鸟类自然保护区采用样方法研究了紫水鸡 (Porphyrio porphyrio) 的冬季受食地选择。研究结果显示, 紫水鸡在冬季对受食地植被具有明显的选择性, 偏爱水葱（Scirpus tabernaemontani）。对 62 个受食地样方 18 个因子的主成分分析表明, 草本密度、草本均高、草本盖度、灌木种 类、水质和水位这 6 个因子是冬季受食地选择的主要因子。判别分析结果表明, 枯高、动物性食物丰富度、距大 道距离、草本种类、距小道距离、距所处水面边缘距离是区别冬季受食地和对照地样方的主要参数, 判别准确率 可达 78.1\%。紫水鸡冬季多选择在水葱或芦苇（Phragmites communis）等草本植被生长良好、具有特定水位或水 质及中等程度的人为干扰的地方受食。因此, 该地区冬季受食地的恢复和保护对紫水鸡的保护有着重要的意义。

关键词: 紫水鸡; 受食地选择; 主成分分析; 判别分析

中图分类号: Q958.1 文献标识码: A 文章编号: 0254-5853-(2008)03-0291-06

\section{Feeding Site Selection of Purple Swamphen in Winter}

\author{
HU Jun-hua ${ }^{1,2,3,4}$, HU Hui-jian ${ }^{2, *}$, YANG Dao-de ${ }^{3}$, JIANG Zhi-gang ${ }^{1}$ \\ (1. Institute of Zoology, Key Lab of Animal Ecology and Conservation, the Chinese Academy of Sciences, Beijing 100101, China; \\ 2. Guangdong Public Lab for Wildlife Conservation \& Wise Use, South China Institute of Endangered Animals, Guangzhou 510260, China; \\ 3. Central South University of Forestry and Technology, Changsha 410004, China; \\ 4. Graduate School of the Chinese Academy of Sciences, Beijing 100049, China)
}

\begin{abstract}
From November 2006 to February 2007, the characteristics of the feeding sites of Purple Swamphen (Porphyrio porphyrio) in winter were studied in Guangdong Haifeng Avian Natural Reserve, by sampling methods. The results showed that there was obvious selectivity of the vegetation in the feeding sites during winter, and Scirpus tabernaemontani was the preferred flora. We found that the main selection factors at the feeding sites in winter using the principal component analysis of 18 factors at 62 feeding sites, were the density of herbage, the height of herbage, the canopy of herbage, the shrub species, the water quality and the water level. In addition, we found that the main selection factors at the feeding sites using the Step DA between feeding sites and control samples plots, were the height of hay, the abundance of animal foods, the distance to the road, the herbage species, the distance to the byway and the distance to the water's edge. The veracity to distinguish was $78.1 \%$. Purple Swamphen preferred to feed in areas that had more Scirpus tabernaemontani or Phragmites communis, special water levels or water quality and medium interference from humans. Thus, restoration and protection of the extant feeding sites in winter are important for the conservation of Purple Swamphen.
\end{abstract}

Key words: Purple Swamphen (Porphyrio porphyrio); Feeding-site selection; Principal component analysis; Step discriminant analysis

食物和隐蔽条件是影响鸟类生境利用的基本因 素 (Root, 1967)，而冬季是鸟类一年当中最为危险 和脆弱的时期 (Hafner, 1997)。鸟类冬季受食不仅
是为了度过寒冷的冬季, 还要为即将开始的繁殖储 备能量。因此, 冬季受食地的选择对整个种群的稳 定和发展具有重要的意义（Ding, 2004）。

收稿日期: 2007-12-15; 接受日期: 2008-04-15

基金项目: 广东省科学院台站基金(05-07); 广东科技攻关（2005A20901006）

*通讯作者 (Corresponding author), E-mail: huhj@gdei.gd.cn, Tel: 020-84191955

第一作者简介: 男, 博士研究生, 研究方向为动物生态学与保护生物学。E-mail: hujunhua1982@163.com 
紫水鸡（Porphyrio porphyrio）广泛分布在旧大 陆热带地区, 见于地中海沿岸、非洲、亚洲及澳大 利亚 (Cramp \& Simmons, 1980; Del Hoyo et al, 1996）。未被列入全球受胁物种（Del Hoyo et al, 1996）。但是, 分布于地中海西部和南大西洋地区 的指名亚种 (P.p.porphyrio) 已列为稀有物种（欧 洲）(Tucker \& Heath，1994)及濒危物种（葡萄牙）

（Cabral et al, 1990）。在中国, 紫水鸡仅分布于云 南南部和西北部（留鸟）、福建、广东、广西、四 川等少数地区(Yang et al, 1995; Wang et al, 2006)。 国内有少量关于紫水鸡种群现状的报道 (Zhang \& Zhou 1985; Gao \& Jiang, 1999, Hu et al, 2006; Hu et al, 2007）, 但未有涉及冬季受食地的研究。为此, 2006 年 11 月至 2007 年 2 月, 我们在广东海丰鸟类 自然保护区进行了紫水鸡冬季受食地选择的研究。

\section{1 研究区域和方法}

广东海丰鸟类自然保护区位于中国沿海地区的 广东省海丰县境内。保护区自然概况见 $\mathrm{Hu}$ (2006, 2007）。本研究范围是保护区内东关联安围保护站 的核心区。东关联安围保护站 $\left(22^{\circ} 53^{\prime} 22^{\prime \prime}-22^{\circ} 50^{\prime}\right.$ $29^{\prime \prime} \mathrm{N}, 115^{\circ} 19^{\prime} 30^{\prime \prime}-115^{\circ} 11^{\prime} 41^{\prime \prime} \mathrm{E}$ ), 面积 $4501.9 \mathrm{hm}^{2}$, 占保护区总面积的 $38.84 \%$ 。主要植被有水憼

( Scirpus tabernaemontani)、芦苇 (Phragmites communis）及残存的红树林等。

2006 年 11 月-2007 年 2 月, 采用直接观察法 确定紫水鸡的冬季受食地位置。把观察到有紫水鸡 受食活动, 并有新鲜被豚食痕迹的植物枝叶和豚食 中被遗弃的枝叶碎屑视为冬季受食地; 把附近无受 食痕迹处作为对照地。随机选取 $1 \mathrm{~m} \times 1 \mathrm{~m}$ 的受食地、 对照地样方分别为 62 和 84 个。调查以下 18 个参 数：植被类型（分水葱从、芦苇从、灌木丛、水葱 从和芦苇从混杂、芦苇从和灌木丛混杂、水䓤从和 杂草从混杂等); 水位; 距水面边缘距离 (指从样 方到所在生境水面边缘的最近距离); 距大道距离 (指从样方到经常有机动车行驶的道路间距离); 距小道距离 (指从样方到经常有人类活动但没机动 车行驶的道路间距离); 距最近居民点距离 (指从 样方到附近最近居民点间的距离); 灌木种类; 灌 木密度; 灌木盖度; 灌木均高; 草本种类; 草本密 度; 草本盖度; 草本均高; 动物性食物丰富度 [指样 方内动物性食物的丰富程度, 分为缺乏 (1)、一般
（2）、丰富（3）三类]; 枯青比（指样方内枯萎植 被与新鲜植被之间的比例); 枯高（指样方内枯萎 植被的高度); 水质[分为好 (1)、一般 (2)、差 (3) 三类]。为便于量化分析受食地选择, 对样方的植被 类型进行编码: 水葱 (1)、芦苇 (2)、杂灌 (3)、 卤蒝 (4)、水葱和芦苇 (5)、水葱和杂草 $(6)$ 、蓱 类 (7)、杂草 (8)、芦苇和杂草 $(9)$ 、水稻 $(10)$ 、 水葫芦 (11)。数据采用 SPSS 12.0 for Windows 软 件进行统计分析。用主成分分析法对冬季受食地的 特征进行因子分析, 再用 Mann-Whitney U-tests 非 参数检验法和逐步判别法比较冬季受食地和对照 地样方。

\section{2 结 果}

\section{1 不同植被类型的受食地比较}

分布在不同植被类型中的冬季受食地样方各变 量均值和标差见表 1 。其中, 有 27 个受食地样方位 于单一的水苟植被内, 占受食地样方的 $43.5 \%$ 。

\section{2 受食地特征的主成分分析}

对冬季受食地的 18 个变量进行主成分分析（表 2)。结果显示, 特征值大于 1.0 的主成分因子共有 5 个, 其累计贡献率达到 $75.66 \%$, 说明这 5 个主成 分包含了所统计 18 个变量的大部分信息。

第 1 主成分贡献率最高 (为 $36.23 \%$ ), 说明草 本密度、草本均高和草本盖度这 3 个有关草本植被 特征的因子是冬季受食地选择的主要因子; 第 2 个 主成分和第 3 个主成分的贡献率较一致, 分别为 $12.92 \%$ 和 $12.61 \%$, 说明灌木种类、水质和水位是冬 季受食地选择的次主要因子; 第 4 个主成分和第 5 个主成分的贡献率较低, 分别为 $7.84 \%$ 和 $6.03 \%$, 说明与所处水面边缘的距离、草本植被的种类和与 距小道距离是次要因子。

提取这 5 个主成分进行更深入的分析, 经平均 正交旋转后的主成分负荷矩阵见表 3 。其中，第 1 主成分与草本密度、草本均高和草本盖度相关系数 都较大 (均大于 0.84 )。这 3 个因子都体现出草本 植被的隐蔽程度, 反映了紫水鸡冬季偏爱在草本植 被密集、隐蔽性好的地方受食; 对第 2 主成分影响 较大的是灌木种类, 说明紫水鸡冬季受食地选择中 灌木种类起着一定作用, 这与实际观察常见其在卤 偋植被受食相符; 第 3 主成分中水质和水位的影响 最大, 共同反映出紫水鸡冬季受食时对水的要求; 
表 1 不同植被类型紫水鸡冬季受食地比较 (均值土标差)

Tab. 1 Comparison among feeding sites of different vegetations of Porphyrio porphyrio in winter (Mean $\pm S D$ )

\begin{tabular}{|c|c|c|c|c|c|c|}
\hline 变量 Variable & $1(n=27)$ & $2(n=9)$ & $3(n=13)$ & $4(n=6)$ & $5(n=5)$ & $6(n=2)$ \\
\hline 水位 Water level $(\mathrm{cm})$ & $20.00 \pm 4.30$ & $21.44 \pm 6.83$ & $14.08 \pm 5.63$ & $24.83 \pm 7.93$ & $12.00 \pm 12.00$ & $0.00 \pm 0.00$ \\
\hline $\begin{array}{l}\text { 距水面边缘距离 } \\
\text { Distance to water edge (m) }\end{array}$ & $6.30 \pm 1.97$ & $1.00 \pm 0.17$ & $2.23 \pm 0.79$ & $23.50 \pm 10.47$ & $2.20 \pm 0.97$ & $82.50 \pm 2.50$ \\
\hline $\begin{array}{l}\text { 距大道距离 } \\
\text { Distance to road (m) }\end{array}$ & $88.96 \pm 23.71$ & $151.67 \pm 77.39$ & $77.15 \pm 15.39$ & $85.00 \pm 15.65$ & $272.00 \pm 91.71$ & $132.50 \pm 2.50$ \\
\hline $\begin{array}{l}\text { 距小道距离 } \\
\text { Distance to byway (m) }\end{array}$ & $51.93 \pm 4.95$ & $44.11 \pm 5.40$ & $59.46 \pm 5.68$ & $39.00 \pm 10.94$ & $53.00 \pm 11.13$ & $70.00 \pm 0.00$ \\
\hline $\begin{array}{l}\text { 距最近居民点距离 } \\
\text { Distance to nearest house (m) }\end{array}$ & $116.11 \pm 12.86$ & $83.33 \pm 15.46$ & $64.62 \pm 39.29$ & $90.83 \pm 31.18$ & $123 \pm 33.82$ & $32.5 \pm 2.5$ \\
\hline 灌木种类 Shrub species & $0.00 \pm 0.00$ & $0.00 \pm 0.00$ & $3.92 \pm 0.40$ & $0.00 \pm 0.00$ & $3.60 \pm 0.24$ & $0.00 \pm 0.00$ \\
\hline $\begin{array}{l}\text { 灌木密度 } \\
\left.\text { Density of shrub (ind } / \mathrm{m}^{2}\right)\end{array}$ & $0.00 \pm 0.00$ & $0.00 \pm 0.00$ & $156.85 \pm 56.58$ & $0.00 \pm 0.00$ & $93.60 \pm 43.22$ & $0.00 \pm 0.00$ \\
\hline 灌木均高 Height of shrub (cm) & $0.00 \pm 0.00$ & $0.00 \pm 0.00$ & $117.85 \pm 13.20$ & $0.00 \pm 0.00$ & $126.00 \pm 18.02$ & $0.00 \pm 0.00$ \\
\hline 灌木盖度 Canopy of shrub (\%) & $0.00 \pm 0.00$ & $0.00 \pm 0.00$ & $80.77 \pm 2.82$ & $0.00 \pm 0.00$ & $61.00 \pm 7.48$ & $0.00 \pm 0.00$ \\
\hline 草本种类 Herbage species & $1.00 \pm 0.00$ & $2.00 \pm 0.00$ & $0.00 \pm 0.00$ & $5.00 \pm 0.00$ & $2.00 \pm 0.00$ & $6.00 \pm 0.00$ \\
\hline $\begin{array}{l}\text { 草本密度 } \\
\text { Density of herbage (ind } / \mathrm{m}^{2} \text { ) }\end{array}$ & $902.07 \pm 66.57$ & $818.22 \pm 151.92$ & $0.00 \pm 0.00$ & $999.33 \pm 151.50$ & $560.00 \pm 161.61$ & $558.00 \pm 6.00$ \\
\hline $\begin{array}{l}\text { 草本均高 } \\
\text { Height of herbage (cm) }\end{array}$ & $123.22 \pm 7.90$ & $160.22 \pm 19.71$ & $0.00 \pm 0.00$ & $120.83 \pm 13.62$ & $131.20 \pm 35.01$ & $89.00 \pm 4.00$ \\
\hline $\begin{array}{l}\text { 草本盖度 } \\
\text { Canopy of herbage (\%) }\end{array}$ & $72.22 \pm 4.00$ & $70.56 \pm 6.53$ & $0.00 \pm 0.00$ & $77.50 \pm 5.59$ & $50.00 \pm 10.84$ & $72.50 \pm 2.50$ \\
\hline $\begin{array}{l}\text { 动物性食物丰富度 } \\
\text { Abundance of animal foods }\end{array}$ & $1.26 \pm 0.14$ & $1.00 \pm 0.00$ & $1.15 \pm 0.10$ & $1.67 \pm 0.33$ & $1.00 \pm 0.00$ & $1.00 \pm 0.00$ \\
\hline $\begin{array}{l}\text { 枯青比 } \\
\text { Ratio between hay and green }\end{array}$ & $0.48 \pm 0.01$ & $0.05 \pm 0.02$ & $0.00 \pm 0.00$ & $0.05 \pm 0.00$ & $0.07 \pm 0.02$ & $0.04 \pm 0.00$ \\
\hline 枯高 Height of hay (cm) & $84.19 \pm 15.23$ & $100.78 \pm 17.39$ & $0.00 \pm 0.00$ & $100.33 \pm 13.18$ & $89.00 \pm 12.10$ & $8.50 \pm 0.50$ \\
\hline 水质 Water quality & $1.81 \pm 0.26$ & $1.67 \pm 0.53$ & $0.85 \pm 0.37$ & $2.33 \pm 0.49$ & $0.40 \pm 0.40$ & $0.00 \pm 0.00$ \\
\hline
\end{tabular}

1: 水葱丛 Scirpus tabernaemontani; 2: 芦苇丛 Phragmites communis; 3: 灌木丛 Shrub; 4: 水葱丛和芦苇丛混杂 Scirpus tabernaemontani \& Phragmites communis miscellaneous; 5: 芦苇丛和灌木丛混杂 Phragmites communis \& Shrub miscellaneous; 6: 水葱丛和杂草丛混杂 Scirpus tabernaemontani \& Weeds miscellaneous.

第 4 主成分与所处水面边缘的距离和草本植被的种 类密切相关, 说明紫水鸡喜欢在离水面边缘一定距 离受食, 且偏爱特定的某些草本植物; 第 5 主成分 与距小道距离的相关系数最大 (达 0.94 ), 表明紫 水鸡冬季受食中对与小道的距离有着明显的选择 性, 这反映出人为活动对其产生的影响。

\section{3 受食地选择的判别分析}

将紫水鸡冬季受食地样方与对照地样方进行对 比分析。结果表明, 两者在距小道距离、植被类型、 距大道距离、草本均高、动物性食物丰富度、枯高 6 个变量上存在显著或极显著差异（表 4)。

逐步判别分析结果表明, 枯高、动物性食物丰 富度、距大道距离、草本种类、距小道距离、距所 处水面边缘距离是区别受食地和对照地样方的 6 个 主要变量 (表 5)。Fisher 线性判别函数系数显示, 这 6 个变量对受食地和对照地样方进行判别分析的
准确率可达 $78.1 \%$ 。

\section{3 讨 论}

紫水鸡是一种杂食性鸟类, 但主要以植物为食, 吃水生和半水生植物的嫩枝、叶、根、茎、花和种 子; 动物性食物占小部分 (Wang et al, 2006)。在东 关联安围对紫水鸡冬季受食地选择分析结果表明, 水葱从是紫水鸡冬季受食时的首选植被类型。根据 野外观察, 可能是由于冬季食物相对匮乏, 水苟 (主 要是根部) 是紫水鸡的最重要食物来源。因此, 充 足的水葱植被对于紫水鸡度过冬季, 并在来年取得 繁殖成功有着积极作用。

从 18 个变量的主成分分析可看出, 紫水鸡倾向 于某一特定密度、高度和盖度的水苟或芦苇从中受 食, 这可能与隐蔽程度和逃避敌害有关。草本植被 的特征值是其冬季受食地选择的主要因素, 水和灌 
表 2 紫水鸡冬季受食地特征的主成分分析 $(n=62)$

Tab. 2 The principal component analysis on the characteristics of feeding sites of Porphyrio porphyrio in winter

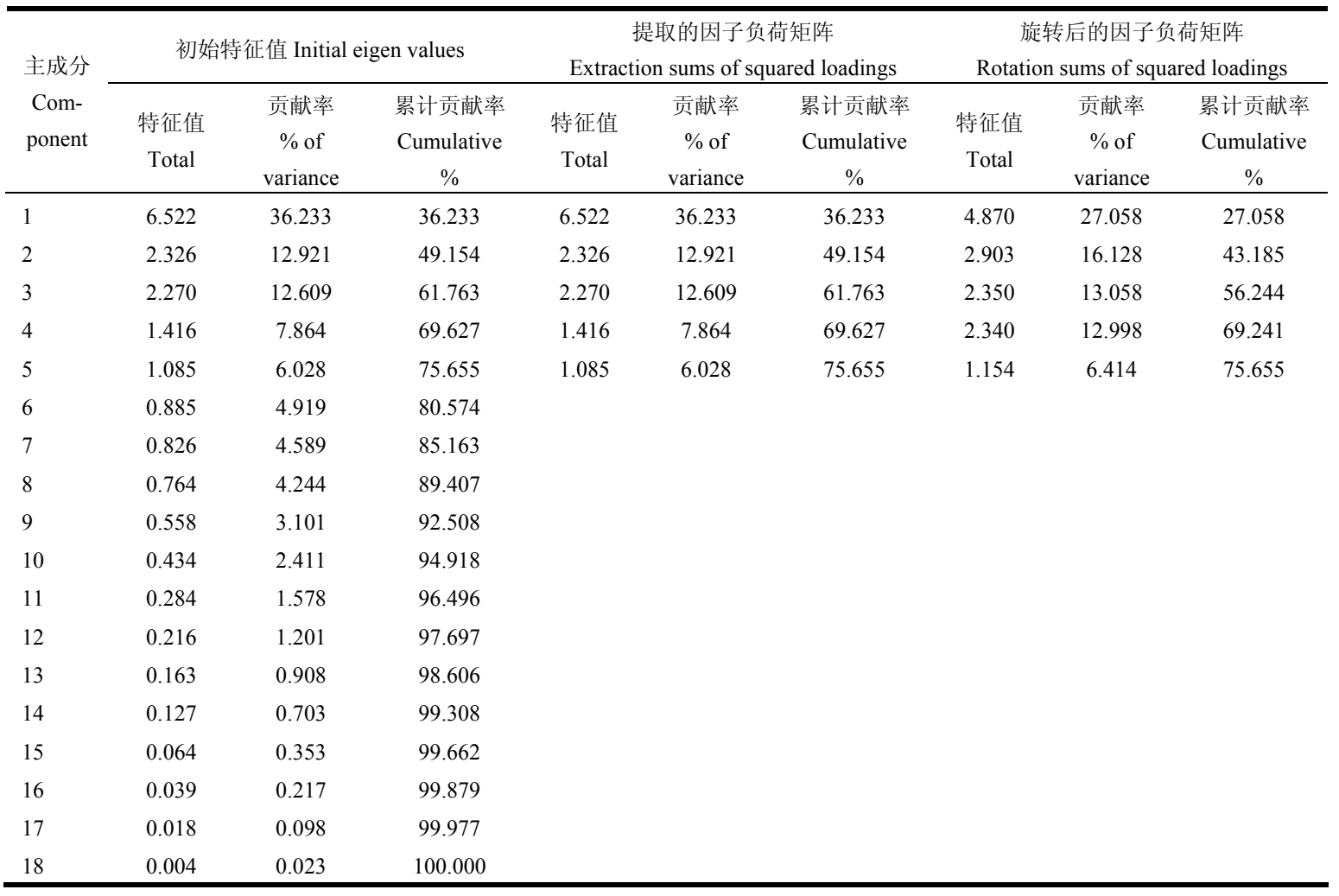

表 3 旋转后的主成分负荷矩阵

Tab. 3 Rotated component matrix

\begin{tabular}{|c|c|c|c|c|c|}
\hline \multirow{2}{*}{$\begin{array}{c}\text { 变量 } \\
\text { Variable }\end{array}$} & \multicolumn{5}{|c|}{ 主成分 Component } \\
\hline & 1 & 2 & 3 & 4 & 5 \\
\hline 植被类型 Vegetation type & -0.091 & 0.639 & -0.281 & 0.671 & 0.009 \\
\hline 水位 Water level & 0.199 & 0.201 & 0.769 & -0.087 & 0.107 \\
\hline 距水面边缘距离 Distance to water edge & -0.025 & -0.196 & -0.011 & 0.875 & 0.117 \\
\hline 距大道距离 Distance to road & 0.009 & 0.072 & -0.603 & 0.054 & -0.017 \\
\hline 距小道距离 Distance to byway & -0.104 & 0.031 & 0.096 & 0.042 & 0.944 \\
\hline 距最近居民点距离 Distance to nearest house & 0.539 & 0.117 & -0.400 & -0.465 & 0.216 \\
\hline 灌木种类 Shrub species & -0.519 & 0.816 & -0.025 & -0.117 & 0.053 \\
\hline 灌木密度 Density of shrub & -0.271 & 0.750 & 0.202 & -0.147 & -0.113 \\
\hline 灌木均高 Height of shrub & -0.518 & 0.625 & -0.299 & -0.083 & 0.171 \\
\hline 灌木盖度 Canopy of shrub & -0.656 & 0.672 & -0.147 & -0.109 & 0.110 \\
\hline 草本种类 Herbage species & 0.469 & -0.004 & -0.077 & 0.801 & -0.142 \\
\hline 草本密度 Density of herbage & 0.844 & -0.288 & 0.082 & -0.023 & -0.010 \\
\hline 草本均高 Height of herbage & 0.875 & -0.174 & -0.005 & -0.026 & -0.191 \\
\hline 草本盖度 Canopy of herbage & 0.882 & -0.335 & 0.114 & 0.085 & -0.095 \\
\hline 动物性食物丰富度 Abundance of animal foods & -0.085 & -0.215 & 0.396 & 0.413 & 0.254 \\
\hline 枯青比 Ratio between hay and green & 0.731 & -0.197 & 0.048 & 0.121 & 0.052 \\
\hline 枯高 Height of hay & 0.664 & -0.088 & 0.092 & -0.010 & -0.001 \\
\hline 水质 Water quality & 0.140 & -0.086 & 0.895 & 0.067 & -0.024 \\
\hline
\end{tabular}


表 4 紫水鸡冬季受食地样方与对照样方的比较

Tab. 4 Comparison between feeding sites and control sample plots of Porphyrio porphyrio in winter

\begin{tabular}{|c|c|c|c|c|}
\hline \multirow{2}{*}{ 变量 Variable } & 受食地 Feeding site $(n=62)$ & 对照地 Control site ( $n=84$ ) & \multirow{2}{*}{$\begin{array}{c}\text { Mann- } \\
\text { Whitney } \\
\text { U -test } Z\end{array}$} & \multirow{2}{*}{$\begin{array}{c}\text { 显著性 } \\
\text { Sig. }\end{array}$} \\
\hline & 均值 \pm 标差 $M e a n \pm S D$ & 均值 \pm 标差 $M e a n \pm S D$ & & \\
\hline 植被类型 Vegetation type & $2.34 \pm 1.48$ & $3.74 \pm 2.79$ & -2.961 & $0.003^{* *}$ \\
\hline 水位 Water level & $18.15 \pm 21.26$ & $17.77 \pm 22.48$ & -0.065 & 0.948 \\
\hline 距水面边缘距离 Distance to water edge & $8.47 \pm 18.01$ & $4.68 \pm 13.95$ & -0.627 & 0.531 \\
\hline 距大道距离 Distance to road & $111.37 \pm 141.19$ & $53.07 \pm 61.85$ & -3.457 & $0.001^{* *}$ \\
\hline 距小道距离 distance to byway & $51.79 \pm 23.37$ & $69.60 \pm 42.97$ & -2.194 & $0.028^{*}$ \\
\hline 距最近居民点距离 Distance to nearest house & $95.97 \pm 62.84$ & $100.39 \pm 50.92$ & -1.327 & 0.185 \\
\hline 灌木种类 Shrub species & $1.11 \pm 1.87$ & $1.13 \pm 1.80$ & -0.319 & 0.749 \\
\hline 灌木密度 Density of shrub & $40.44 \pm 114.45$ & $67.11 \pm 113.38$ & -0.550 & 0.582 \\
\hline 灌木均高 Height of shrub & $34.87 \pm 59.81$ & $39.86 \pm 66.53$ & -0.255 & 0.799 \\
\hline 灌木盖度 Canopy of shrub & $21.85 \pm 35.33$ & $22.62 \pm 36.40$ & -0.037 & 0.970 \\
\hline 草本种类 Herbage species & $1.56 \pm 1.58$ & $3.12 \pm 3.62$ & -1.874 & 0.061 \\
\hline 草本密度 Density of herbage & $671.48 \pm 482.61$ & $546.74 \pm 435.45$ & -1.584 & 0.113 \\
\hline 草本均高 Height of herbage & $102.06 \pm 68.54$ & $65.33 \pm 57.47$ & -3.323 & $0.001^{* *}$ \\
\hline 草本盖度 Canopy of herbage & $55.56 \pm 34.08$ & $51.06 \pm 38.25$ & -0.187 & 0.852 \\
\hline 动物性食物丰富度 Abundance of animal foods & $1.21 \pm 0.58$ & $0.95 \pm 0.21$ & -3.622 & $0.000^{* *}$ \\
\hline 枯青比 Ratio between hay and green & $0.04 \pm 0.03$ & $0.03 \pm 0.03$ & -1.183 & 0.237 \\
\hline 枯高 Height of hay & $68.45 \pm 68.37$ & $31.57 \pm 30.25$ & -3.945 & $0.000^{* *}$ \\
\hline 水质 Water quality & $1.47 \pm 1.42$ & $1.48 \pm 1.31$ & -0.215 & 0.830 \\
\hline
\end{tabular}

${ }^{*} P<0.05 ;{ }^{* *} P<0.01$.

表 5 紫水鸡冬季受食地样方与对照样方的逐步判别分析结果

Tab. 5 Results of step DA between feeding sites and control sample plots of Porphyrio porphyrio in winter

\begin{tabular}{|c|c|c|c|c|c|}
\hline $\begin{array}{l}\text { 步骤 } \\
\text { Step }\end{array}$ & $\begin{array}{c}\text { 变量 } \\
\text { Variable }\end{array}$ & $\begin{array}{l}\text { 耐受系数 } \\
\text { Tolerance }\end{array}$ & $\begin{array}{c}F \text { 值变化 } \\
F \text { to Remove }\end{array}$ & $\begin{array}{c}\text { Wilks’ } \lambda \text { 值 } \\
\text { Wilks' Lambda }\end{array}$ & $\begin{array}{l}\text { 显著性 } \\
\text { Sig. }\end{array}$ \\
\hline 1 & 枯高 Height of hay & 1.000 & 19.346 & & 0.000 \\
\hline \multirow[t]{2}{*}{2} & 枯高 Height of hay & 0.999 & 16.446 & 0.911 & \\
\hline & 动物性食物丰富度 Abundance of animal foods & 0.999 & 11.345 & 0.882 & 0.000 \\
\hline \multirow[t]{3}{*}{3} & 枯高 Height of hay & 0.997 & 16.116 & 0.831 & \\
\hline & 动物性食物丰富度 Abundance of animal foods & 0.982 & 13.477 & 0.817 & \\
\hline & 距大道距离 Distance to road & 0.982 & 13.380 & 0.817 & 0.000 \\
\hline \multirow[t]{4}{*}{4} & 枯高 Height of hay & 0.990 & 17.122 & 0.784 & \\
\hline & 动物性食物丰富度 Abundance of animal foods & 0.971 & 9.888 & 0.748 & \\
\hline & 距大道距离 Distance to road & 0.966 & 15.380 & 0.775 & \\
\hline & 草本种类 Herbage species & 0.964 & 9.580 & 0.746 & 0.000 \\
\hline \multirow[t]{5}{*}{5} & 枯高 Height of hay & 0.975 & 19.131 & 0.746 & \\
\hline & 动物性食物丰富度 Abundance of animal foods & 0.970 & 9.947 & 0.703 & \\
\hline & 距大道距离 Distance to road & 0.956 & 11.659 & 0.711 & \\
\hline & 草本种类 Herbage species & 0.956 & 10.644 & 0.706 & \\
\hline & 距小道距离 Distance to byway & 0.961 & 9.066 & 0.699 & 0.000 \\
\hline \multirow[t]{6}{*}{6} & 枯高 Height of hay & 0.967 & 20.103 & 0.730 & \\
\hline & 动物性食物丰富度 Abundance of animal foods & 0.827 & 4.117 & 0.657 & \\
\hline & 距大道距离 Distance to road & 0.946 & 9.613 & 0.682 & \\
\hline & 草本种类 Herbage species & 0.756 & 14.818 & 0.706 & \\
\hline & 距小道距离 Distance to byway & 0.914 & 11.313 & 0.690 & \\
\hline & 距水面边缘距离 Distance to water edge & 0.692 & 4.100 & 0.656 & 0.000 \\
\hline
\end{tabular}


木种类因素是次重要因素。冬季受食地的水位和水 质, 决定着紫水鸡对冬季受食地内食物的可利用程 度, 过深的水位或没有水都会影响紫水鸡的受食; 水质的好坏在某种程度上影响紫水鸡对植物性食 物的选择。干扰因素是冬季受食地选择的次要因 素, 但也是不能忽视的重要因素。冬季受食地与附 近大、小道路之间的距离与人为干扰的强度有密切 联系，制约着紫水鸡对冬季受食地的选择。

紫水鸡冬季受食地样方与对照地样方对比分析 的结果表明, 两者在距小道距离、植被类型、距大 道距离、草本均高、动物性食物丰富度、枯高方面 存在显著或极显著差异。通过判别分析, 枯高、动 物性食物丰富度、距大道距离、草本种类、距小道 距离、距所处水面边缘距离是区别冬季受食地和对

\section{参考文献:}

Cabral MJ, Magalhăes CP, Oliveira ME, Romăo C. 1990. Livro Vermelho dos Vertebrados de Portugal. Volume 1 - Mamíferos, Aves, Répteis e Anfíbios. Servico Nacional de Parques, Reservas e Conservacăo da Natureza, Secretaria de Estado do Ambiente e Defesa do Consumidor [M]. Portugal: Lisboa.

Cramp S, Simmons KEL. 1980. The Birds of the Western Palearctic. In: Hawks to Bustards, vol. 2 [M]. Oxford: Oxford University Press.

Del Hoyo J, Elliott A, Sargatal J. 1996. Handbook of the Birds of the World. Vol.3. Hoatzin to Auks [M]. Barcelona: Lynx Edicions, 196-197.

Ding CQ. 2004. Research on the Crested Ibis [M]. Shanghai: Shanghai Scientific \& Technological Education Publishing House, 32-43. [丁长 青. 2004. 朱噮研究. 上海: 上海科技教育出版社, 32-43.]

Gao YR, Jiang GD. 1999. The purple swamphen founded in Guangdong [J]. Chinese Journal of Zoology, 34(1): 38-39. [高育仁, 蒋果丁. 1999. 广东海丰发现紫水鸡. 动物学杂志, 34(1): 38-39.]

Hafner H. 1997. Ecology of wading birds [J]. Colonial Waterbirds, 20: 115-120.

$\mathrm{Hu}$ JH, Yang DD, Hu HJ, Deng JM. 2006. Breeding nests of Purple Swamphen founded in Haifeng, Guangdong [J]. Chinese Journal of Zoology, 41(6): 166-168. [胡军华, 杨道德, 胡慧建, 邓杰明. 2006. 广东海丰发现紫水鸡繁殖巢. 动物学杂志, 41(6): 166-168.]

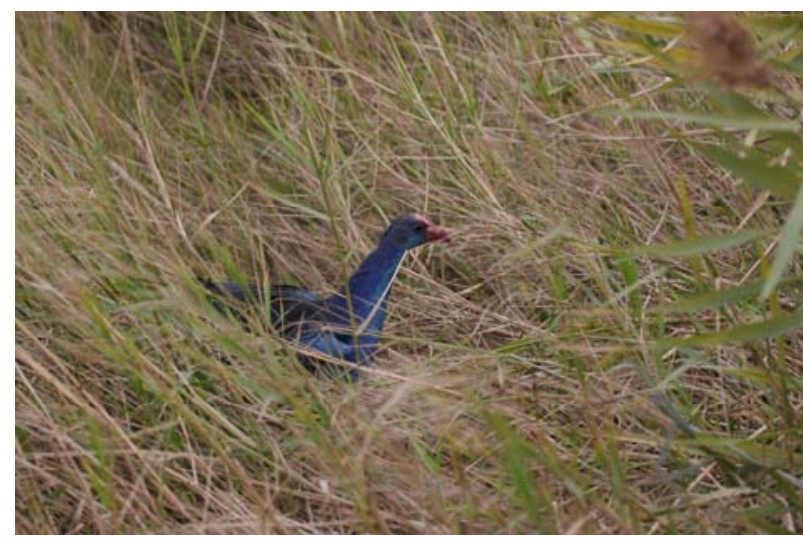

附照 1 紫水鸡（Porphyrio porphyrio） [M]. Cambridge: BirdLife International.
照地样方的最重要参数，判别准确率可达 $78.1 \%$ 。 判别分析和主成分分析得出的结果较为一致，但枯 高和动物性食物丰富度是两者间的主要差别。

总之，紫水鸡冬季多选择在水葱或芦苇等草本 植被生长良好、具有特定水位或水质及中等程度的 人为干扰的地方受食。因此, 对于现有紫水鸡冬季 受食地的恢复和保护对于紫水鸡的保护有着重要 的意义。

致谢：广东海丰鸟类自然保护区全体工作人员 和当地群众在调查期间给予了大力支持; 广东象头 山国家级自然保护区的邓杰明先生和广东南岭国 家级保护区的杨昌腾先生参与了本文的野外调查, 一并表示衷心感谢!

Hu JH, Hu HJ, Yang DD, Deng JM. 2007. Population estimation of Purple Swamphen (Porphyrio porphyrio) in Haifeng, Guangdong [J]. Chinese Journal of Zoology, 42(1): 107-111. [胡军华, 胡慧建, 杨道 德, 邓杰明. 2007. 广东海丰紫水鸡种群密度调查. 动物学杂志, 42(1): 107-111.]

Root RB. 1967. The niche exploitation pattern of the Blue-gray Gnatcatcher [J]. Ecological Monographs, 37, 317-350.

Tucker GM, Heath MF. 1994. Birds in Europe: Their Conservation Status

Wang QS, Ma M, Gao YR, 2006. Fauna Sinica.Aves vol.5.Gruiformes, Charadriiformes and Lariformes [M]. Beijing: Science Press. [王岐山, 马 鸣, 高育仁. 2006. 中国动物志 鸟纲 第五卷 鹤形目 行形目 鸥形目. 北京: 科学出版社.]

Yang L, Wen XJ, Han LX, Yang XJ. 1995. The Avifauna of Yunnan China. vol.I. Non- Passeriformes [M]. Kunming: Yunnan Science and Technology Press. [杨岗, 文贤继, 韩联宪, 杨晓君. 1995. 云南鸟 类志 上卷 非雀形目. 昆明: 云南科技出版社.]

Zhang JJ, Zhou DY. 1985. The Purple Swamphen founded in sichuan firstly [J]. Sichuan Journal of Zoology, (2): 19. [张家驹, 周道远. 1985. 四 川首次发现紫水鸡, 四川动物, (2): 19.] 